\title{
Trunk Muscle Activity in One- and Two-Armed American Kettlebell Swing in Resistance-Trained Men
}

\section{(ㄷ) (1) (8) $\Theta$}

\author{
Authors \\ Vidar Andersen ${ }^{1}$, Marius Steiro Fimland ${ }^{2,}{ }^{\text {, }}$, Atle Saeterbakken ${ }^{1}$
}

\section{Affiliations}

1 Faculty of Education, Arts and Sports, Western Norway University of Applied Sciences, Norway.

2 Department of Neuromedicine and Movement Science, Faculty of Medicine and Health Sciences, Norwegian University of Science and Technology, Trondheim, Norway.

3 Department of Physical Medicine and Rehabilitation, St. Olavs Hospital, Trondheim University Hospital, Trondheim, Norway

Key words unilateral, bilateral, EMG, core, strength, resistance training

$\begin{array}{ll}\text { received } & 17.10 .2018 \\ \text { revised } & 22.02 .2019 \\ \text { accepted } & 25.02 .2019\end{array}$

\section{Bibliography \\ DOI https://doi.org/10.1055/a-0869-7228}

Sports Medicine International Open 2019; 3: E12-E18

(c) Georg Thieme Verlag KG Stuttgart · New York

ISSN 2367-1890

\section{Correspondence}

Mr. Vidar Andersen

Faculty of Education, Arts and Sports, Western Norway University of Applied Sciences,

$\mathrm{Pb} 133$,

Sogndal 6851

Norway

Tel.: + 47/676/76 164, Fax: +47/576/76 333

vidar.andersen@hvl.no

\section{ABSTRACT}

The aim of the study was to compare the one-armed vs. twoarmed American kettlebell swing on trunk muscle activation. Fifteen resistance-trained men performed ten repetitions of both exercises using a 14-kg kettlebell. Surface EMG from the erector spinae, rectus abdominis and external oblique muscles were collected on both sides of the trunk. The erector spinae activation during the one-armed swing was $14-25 \%$ higher on the contralateral compared to the ipsilateral side in both exercises (Cohen's d effect size [ES] $=0.41-0.71, \mathrm{p}<0.001-0.034$ ). Further, the contralateral side was $14 \%$ more activated during the two-armed swing compared to the ipsilateral side during the one-armed swing ( $E S=0.43, p=0.009$ ). For the rectus abdominis muscle, the two-armed swing induced higher activation of the rectus abdominis compared to the one-armed swing on both the contralateral $(40 \%, E S=0.48, p=0.040)$ and ipsilateral side $(59 \%, E S=0.83, p=0.002)$. There were no differences for the external oblique muscle ( $p=0.495-0.662)$. In conclusion, the trunk activation patterns of the two exercises were different, which could be explained by different biomechanics in the two exercises, and could thus have complimentary effects. We recommend that both unilateral and bilateral execution of the American kettlebell swing is included over time.

\section{Introduction}

In recent years, kettlebell training has become a very popular form of resistance-training exercise and it is used for a variety of purposes, from rehabilitation to increasing athletic performance $[4,12,13]$. Studies show that kettlebells can be used to improve 1-RM in back squat [11], and the power output in vertical jump and power clean
[15] among healthy men. Further, Kettlebell training has also led to increased aerobic capacity of female soccer players [7].

Variations of the kettlebell swing are frequently used in kettlebell training. In this exercise, extension of the hip creates an impulse, propagated through the trunk and arms to the kettlebell, which is propelled forward and upward in a swing movement. There 
are two main styles of kettlebell swing: the "Russian," where the upward movement is finished when the kettlebell is in front of the head; and the "American," where it finishes directly above the head.

Previous research has shown that performing resistance exercises unilaterally or bilaterally affects trunk muscle activation differently $[3,16,18]$. This is generally explained by an increase in the activation of the contralateral side during unilateral exercises to avoid postural sway, especially when the weight is lifted in a trajectory lateral to the trunk. Although the scientific evidence is limited, an increase in the activation of the contralateral side during unilateral versus bilateral execution seems to be accompanied by reduced activation on the ipsilateral side [3], but this should be investigated further.

The kettlebell swing can also be performed unilaterally or bilaterally. We previously investigated muscle activation of trunk muscles during the one- versus two-armed Russian kettlebell swing [2]. The results for the upper erector spinae muscle showed that the contralateral side during the one-armed swing was most activated, whereas the ipsilateral side during the same exercise was the least activated. Interestingly, the opposite pattern was found for the rectus abdominis muscle, with the contralateral side during the one-armed swing being inferior to the ipsilateral side during the one-armed swing and both sides during the two-armed swing. However, results from the Russian kettlebell swing cannot necessarily be transferred to the American variant, because the exercises have different kinetics and kinematics. The American swing shows a longer cycle time and higher vertical impulse [5]. Importantly, in the American swing the endpoint of the kettlebell is in a vertical position over the head, which likely inflicts stress on the trunk muscles to avoid hyperextension and / or rotation of the trunk. Further, the trunk muscles are important for the execution because the impulses created by the hip have to be transferred through them to reach the kettlebell.

Therefore, the aim of the study was to compare the muscle activation of the erector spinae, the rectus abdominis and the external oblique muscle on both sides of the trunk between the one- and two-armed American swing. Based on the results from resistance training studies [3] and our previous Russian kettlebell investigation [2], we hypothesized greater activation on the contralateral side of the erector spinae muscle during the one-armed American swing compared to the ipsilateral side in the same exercise, and to the activation obtained during the two-armed American swing. Further, we expected the ipsilateral side during the one-armed swing to have the lowest activation. For the rectus abdominis muscle, we expected the opposite pattern and no difference between sides and exercises for the external oblique muscle.

\section{Material and Methods}

\section{Study overview}

A within-subject crossover design was used to measure the EMG activity of the erector spinae, the rectus abdominis, and the external oblique muscle on both sides of the trunk during 10 repetitions of the one- and two-armed American kettlebell swing using a 14-kg kettlebell in resistance-trained men. Four practice sessions with a certified kettlebell instructor were conducted. The order of the oneand two-armed swing was counterbalanced and randomized.

\section{Participants}

A sample size calculation was performed requesting a power of $80 \%$ and allowing an effect size of 0.7 to be significant at the $5 \%$ level of significance. The value of the effect size was based on two previous studies [2,3]. The calculations showed that a minimum of 12 participants should be recruited to the study.

Fifteen healthy men (age $24.0 \pm 5.2$ years, body mass $78.5 \pm 7.4 \mathrm{~kg}$, stature $180 \pm 7 \mathrm{~cm}$ ) with $5.3 \pm 4.7$ years of resistance training experience, signed up for the study. The subjects had no or little experience with the kettlebell exercises prior to the study. To be included, they had to be free of injuries or pain that would impair execution of the exercises. Further, the subjects agreed to refrain from any resistance exercise in the $72 \mathrm{~h}$ before testing. All participants were informed orally and in writing of the procedures and possible risks of the test and provided written consent before they were included in the study. The study conformed to the latest revision of the Declaration of Helsinki, the ethical guidelines at the Sogn and Fjordane University College, and the ethical standards of the International Journal of Sports Medicine [9]. All appropriate consent pursuant to law was obtained before the start of the study.

\section{Procedures}

In the experimental test session, the participants first cycled on an ergometer cycle or walked on a treadmill for $5 \mathrm{~min}$. Afterwards, they performed one set of one-armed and one set of two-armed American kettlebell swing, each consisting of ten repetitions using a $10-\mathrm{kg}$ kettlebell. The order of the one- and two-armed American swing was the same in the warm-up and the experimental test. In the one-armed American swing, the dominant arm was used, defined as the arm used to throw a ball.

During the first exercise, the participants self-selected the distance between their feet, which was noted and used in all tests. The exercise started with the arm in a horizontal position with a test leader supporting the kettlebell. On the participant's signal, the kettlebell was dropped and the participants performed 11 consecutive repetitions. The first repetition was discarded from the analysis. The repetitions were performed at a self-selected tempo. The participants were instructed to bend their knees slightly, keeping their arms and back straight throughout the movement, and to create the movement using their hip extensors ( $\vee$ Fig. 1 and $\triangleright 2$ ). To ensure the same range of motion between the repetitions and exercises, the upper / end position (vertical arm) was controlled using a rubber band. The band was positioned so the kettlebell touched it when the arm of the participants was vertical. In the lowest position, the back was kept straight. The arms touched the legs causing a natural stop of the swing before starting the upwards movement. All tests were performed with a 14-kg kettlebell (Sportsmaster Pro kettlebell; Sportsmaster, Nesbru, Norway). This weight was chosen after the practice sessions because some of the participants struggled with the technique when using a heavier kettlebell. To assess the perceived effort during the uni- and bilateral swing, we included a perceived exertion scale (Borg CR10-scale ${ }^{\circledR}$ ), which was used immediately after each test. 3 min of rest were given between each test.

A linear encoder (Ergotest Technology AS, Langesund, Norway), placed on the floor between the participant's feet, was used to measure the range of motion, divide the movement into different 
phases and measure the swing time. The upward and the downward movements were each divided into three phases (lower, middle and upper), based on the trajectory of the kettlebell (cm; lower third, middle third, upper third) ( $\triangleright$ Fig. 1 and $\triangleright 2$ ). Commercial software (MuscleLab v8.13, Ergotest Technology AS, Langesund, Norway) was used to identify and analyze the different phases.

\section{Electromyography}

Before placing the gel-coated self-adhesive electrodes (Dri-Stick Silver circular sEMG Electrodes AE-131, NeuroDyne Medical, Cambridge, Massachusetts, USA), the skin was shaved, abraded and washed with alcohol according to the recommendations by SENIAM [10]. The electrodes ( $11 \mathrm{~mm}$ contact diameter and a $2 \mathrm{~cm}$ centerto-center distance) were placed according to the recommendations by SENIAM (www.seniam.org), along the presumed direction of the underlying muscle fibers on the erector spinae muscle (at L1 and $3 \mathrm{~cm}$ lateral to the spinous process), rectus abdominis muscle ( $3 \mathrm{~cm}$ lateral to the umbilicus), and external oblique muscle (approximately $15 \mathrm{~cm}$ from the umbilicus) on both the contralateral and ipsilateral side. The contralateral and ipsilateral sides were defined from the one-armed American swing, but the terms are also used for the two-armed American swing. The raw EMG signal was captured analogously, amplified and filtered (8-600 Hz; fourth-order Butterworth filter) using a preamplifier located close to the sampling point. The preamplifier had a common mode rejection ratio of $100 \mathrm{~dB}$. The EMG signals were root-mean-square (RMS) converted using a hardware circuit network (frequency response $0-600 \mathrm{kHz}$, averaging constant $100 \mathrm{~ms}$, total error $\pm 0.5 \%$ ). Finally, the RMS-converted signal was sampled at $100 \mathrm{~Hz}$ in a continuously moving time window using a 16-bit A/D converter. Commercial software (MuscleLab v8.13, Ergotest Technology AS, Langesund, Norway) was used to analyze the stored EMG data. The mean EMG amplitude obtained during all ten repetitions was used to calculate RMS EMG of the whole test. In addition, each repetition was divided into six phases, the upper, middle and lower phase of the upward and downward movement. The mean EMG amplitude of each phase was used to calculate the RMS values. Finally, to normalize EMG activity, the participants performed three maximal voluntary contractions (MVCs). For the abdominal muscles, two isometric sit-ups were used (straight for the rectus abdominis muscle and diagonally for the external oblique muscle), where participants were held in a sitting position with hip and knee angles of $90^{\circ}$. For the erector spinae muscle, the Biering-Sorenson position was used [19]. The participants laid with their lower body fixated to a bench and their upper body off the bench. The participant was instructed to extend their back while they were held manually in a horizontal position. In all MVCs, the participants were instructed to obtain maximal force and hold it for $3 \mathrm{sec}$. Two attempts were performed for each test and the one with the highest EMG amplitude was used to normalize the EMG signal for the relevant muscle during the American kettlebell swing.

\section{Statistical analyses}

A two-way analysis of variance (ANOVA) with repeated measures was used to assess differences in EMG activity for the different muscles. The two factors were exercise (one-armed- and two-armed American swing) and side (contra- and ipsilateral). When interaction or main effects were detected by ANOVA, paired t-tests with Bonferroni post hoc corrections were used to determine where the differences lay. The analyses were performed for both the whole
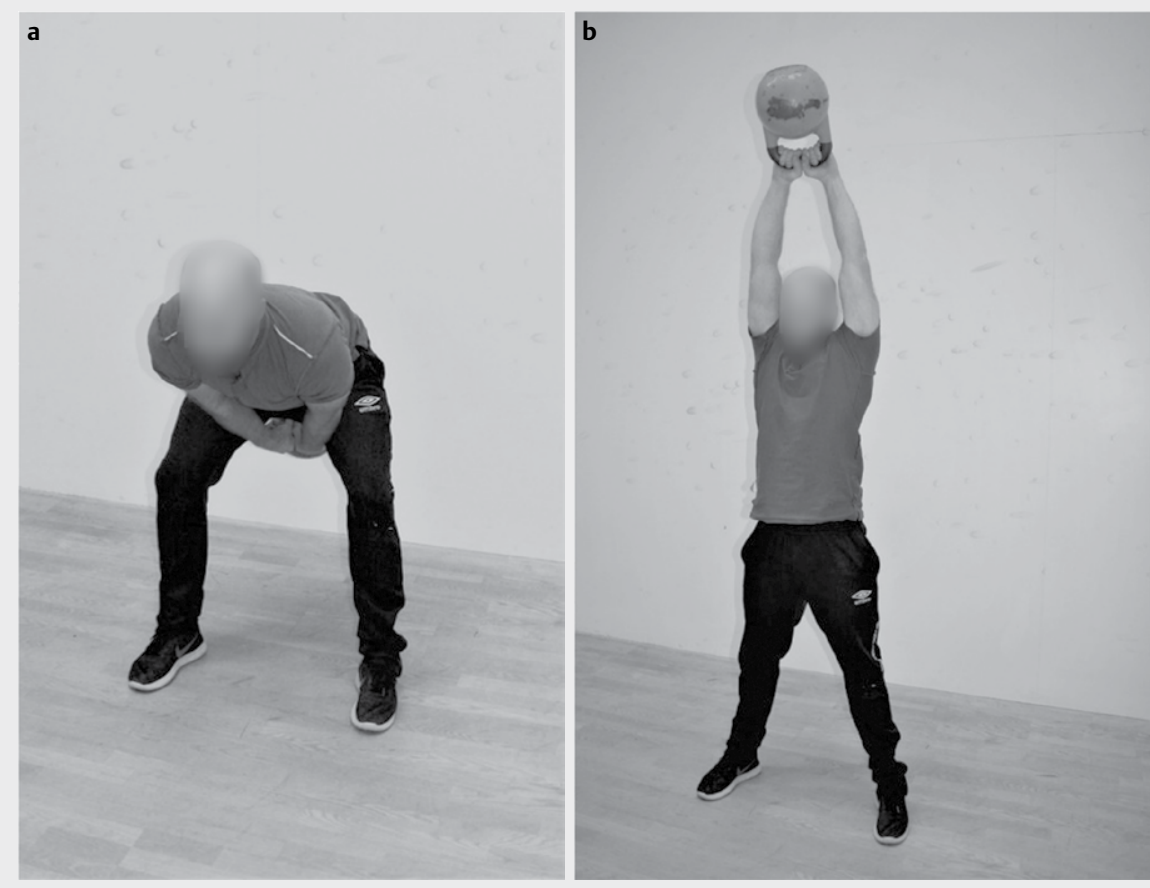

- Fig. 1 Two-armed American kettlebell swing, lower $\mathbf{a}$ and upper $\mathbf{b}$ position. 

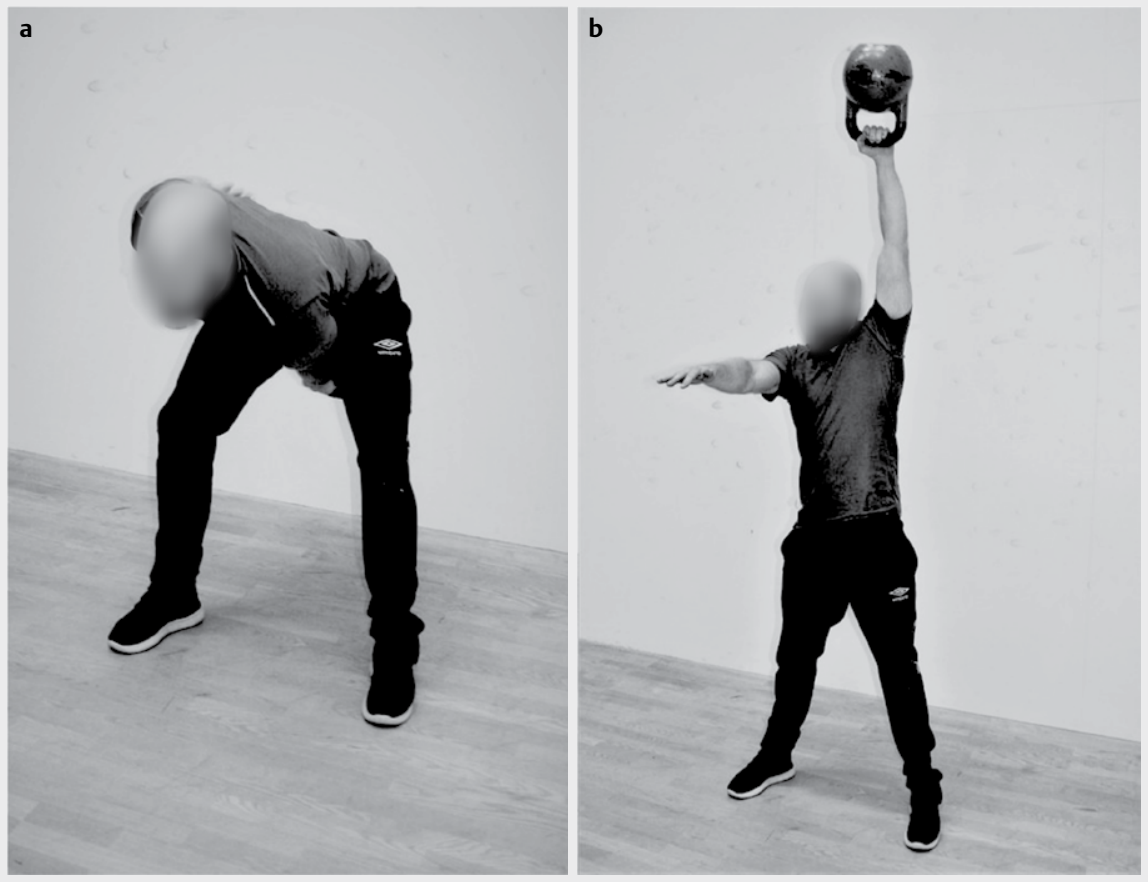

- Fig. 2 One-armed American kettlebell swing, lower $\mathbf{a}$ and upper $\mathbf{b}$ position.

movement and for the different phases. To check for systematic differences in swing time and range of motion between the exercises, a paired t-test was used. Statistical analyses were performed with SPSS version 22.0 (SPSS, Inc. Chicago, IL, USA).

Statistical significance was set to $p \leq 0.05$. All results are presented as mean \pm standard deviation and Cohen `s d effect size (ES). An effect was considered small, medium and large at 0.2, 0.5 and 0.8 , respectively [6].

\section{Results}

When analyzing the mean EMG activity for the whole set of ten repetitions, there was an interaction between exercise and side for the erector spinae muscle $(F=20.208, p=0.006)$. Post hoc tests showed greater activation on the contralateral side during the one-armed swing vs. the ipsilateral side during the one-armed $(25 \%, p<0.001$, $E S=0.71$, Fig. 3a) and two-armed swing (14\%, $p=0.034$, $E S=0.41$ ). Further, the contralateral activation was greater during the two-armed swing vs. the ipsilateral side during the one-armed swing $(14 \%, p=0.009, E S=0.43)$. No other significant differences were observed for the erector spinae muscle $(p=0.115-0.688)$.

When the activation of erector spinae muscle during the upward movement was examined, interaction effects were observed in both the lower $(F=7.223, p=0.018)$ and middle phases $(F=7.098$, $p=0.019)$, and a main effect for exercise was found in the upper phase $(F=9.780, p=0.007)$. During the downward movement, an interaction effect between side and exercise for the erector spinae muscle was observed in the lower phase $(F=18.651, p=0.001)$. There were no interaction nor main effects for the erector spinae muscle in the middle or upper phase of the downward movement $(F=0.391-0.500, p=0.379-0.515)$. Results from the phase-specific post hoc analyses are presented in $\mathbf{F i g .} \mathbf{3 b}$.

For the rectus abdominis muscle activation during the whole set, there was no interaction between exercise and side $(F=2.299$, $p=0.150)$ or main effect for side $(F<0.001, p=0.986)$. However, there was a main effect for exercise $(F=13.900, p=0.002)$. The post hoc analysis showed that the two-armed American swing lead to a 40 and $59 \%$ higher activation of the rectus abdominis muscle compared to the one-armed swing on both the contralateral $(63.8 \pm 45.4$ of MVC vs. $45.3 \pm 29.2 \%, p=0.040$, ES $=0.48$, $>$ Fig. $3 c$ ) and ipsilateral side $(67.2 \pm 37.4$ of MVC vs. $42.2 \pm 20.6 \%, p=0.002$, $E S=0.83)$, respectively.

During the different phases, an interaction effect between side and exercise was observed in the upper phase of the upward movement $(F=5.434, p=0.034)$ and the downward movement $(F=17.852, p=0.001)$. There was no interaction or main effect in the middle or lower phase $(F=0.006-4.185, p=0.059-0.938)$ of either the upward or downward movement. Results from the post hoc analyses are shown in $>$ Fig. $\mathbf{3 d}$.

Regarding the whole set for the external oblique muscle, there were no interaction $(F=0.243, p=0.630$, $>$ Figure $3 E)$ or main effects for exercise $(F=0.491, p=0.495)$ or side $(F=0.199, p=0.662)$.

The trajectory lane $(20 \mathrm{~cm})$ and lifting time $(1.3 \mathrm{sec})$ was longer for the one-armed compared to the two-armed swing ( $<<0.001)$, but the mean lifting velocity (distance / time) was similar ( $1.40 \pm 0.16 \mathrm{vs}$. $1.41 \pm 0.19 \mathrm{~m} / \mathrm{s}, \mathrm{p}<0.972$ ). The rating of perceived exertion was the same for both exercises (one-armed: $4.81 \pm 1.22$, two-armed: $4.75 \pm 1.18)$. 
a

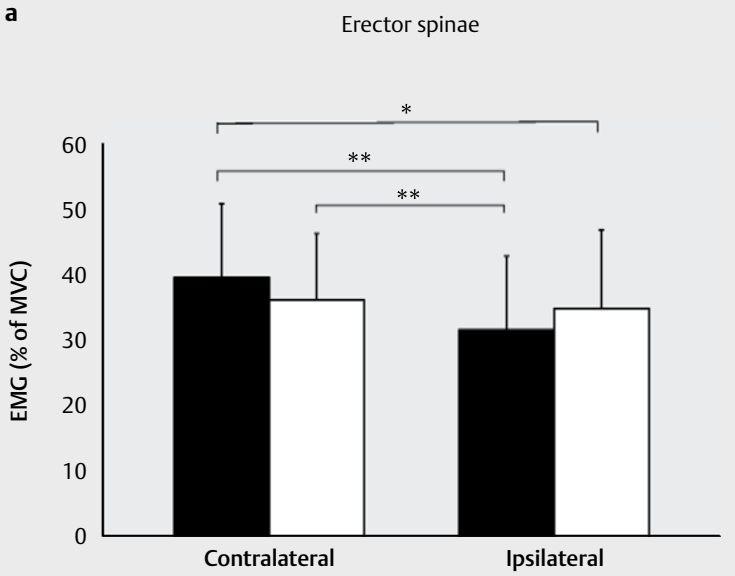

Rectus abdominis

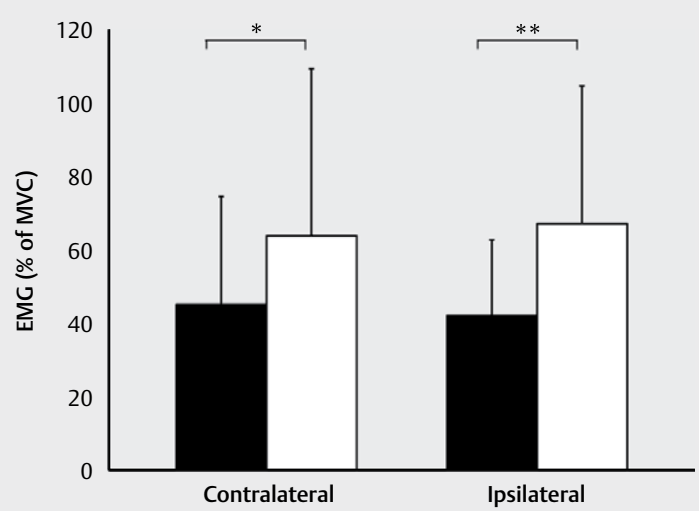

e

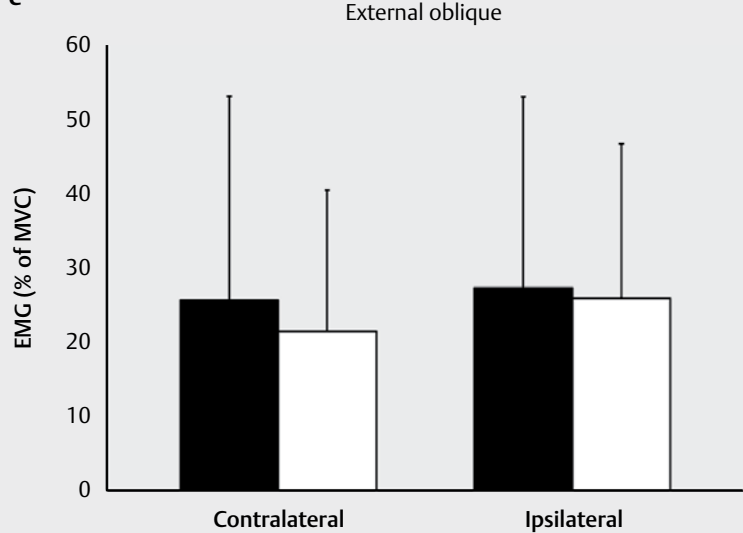

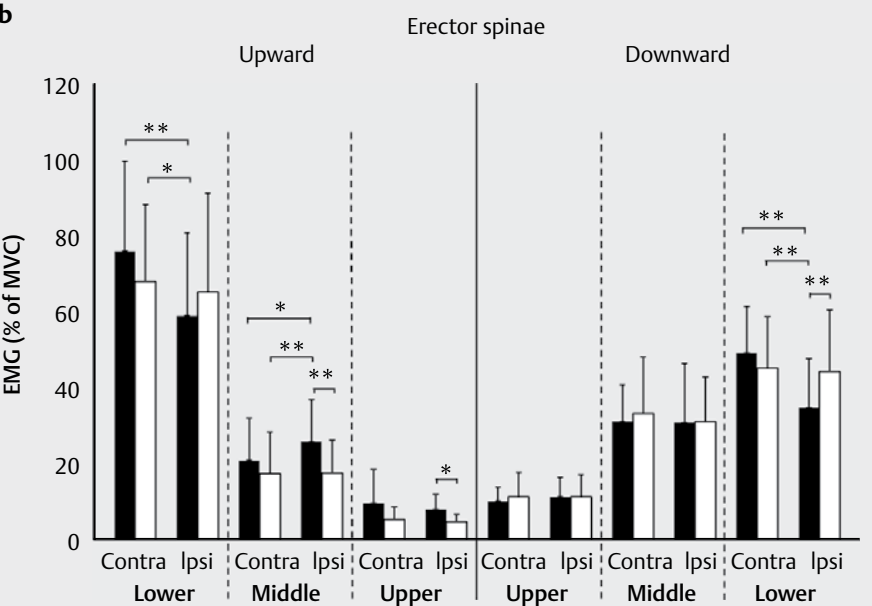

d

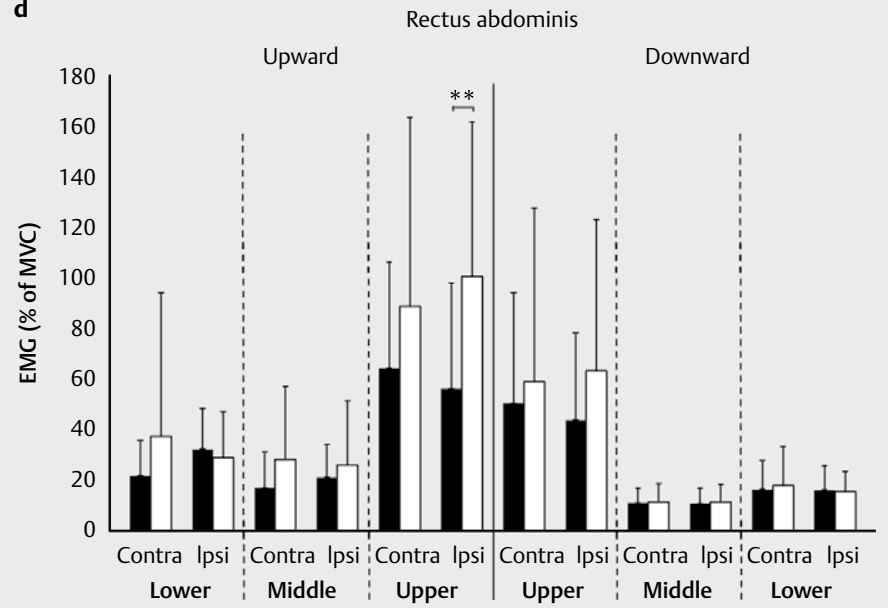

- One arm

$\square$ Two arms

- Fig. 3 Normalized electromyographic (EMG) activation for the whole movement and different phases of the erector spinae muscle (a and $\mathbf{b}$ ), the rectus abdominis muscle ( $\mathbf{c}$ and $\mathbf{d}$ ), and the whole movement of the external oblique muscle $\mathbf{e}$ on the contra- and ipsilateral side during the one- and two-armed American kettlebell swing. Values are means \pm SD. ${ }^{*}{ }^{*} \mathrm{p}<0.01,{ }^{*} \mathrm{p}<0.05$.

\section{Discussion}

The results of this study showed the following: 1) the one-armed American swing induced greater activation of the contralateral side of the erector spinae muscle compared to the ipsilateral side during both one- and two-armed performance; 2) the contralateral side during the two-armed swing was also more activated than the ipsilateral side during the one-armed swing; 3) the two-armed swing provided greater activation of both sides of the rectus abdominis muscle compared to the one-armed swing; and finally 4) there were no differences for the external oblique muscle. 
The main differences between the unilateral and bilateral execution of the American kettlebell swing are: 1) the kettlebell travels in the centerline of the body throughout the movement on bilateral execution, whereas it moves medial to lateral in the upward phase when performed unilaterally (ending above the shoulder instead of the head); and 2) a unilateral swing allows rotation of the trunk, which caused the kettlebell to be moved $20 \mathrm{~cm}$ further through the legs than the two-armed swing ( $\vee$ Fig. 1 and $\triangleright 2$ ). Both these differences could explain why erector spinae muscle activation was highest on the contralateral side and lowest on the ipsilateral side. To resist the lateral-medial movement of the kettlebell in the bottom and rotate back to the neutral position in the beginning of the upward movement with the unilateral swing, the contralateral side of the erector spinae muscle would have to be more activated and the ipsilateral side less activated, just as we also found in our previous investigation of the Russian kettlebell swing [2]. The muscle activation levels were much higher in the lower phase than in the middle and upper phases, and the results from the lower phase showed the same pattern as for the whole movement. Although there were some differences in the pattern of the two other phases, this should have little practical relevance.

Rectus abdominis muscle activation was higher when performing the American swing with both arms compared to one, which we did not expect based on our Russian swing study [2], where the ipsilateral side during the one- and two-armed execution was most activated. However, in the Russian swing the upward movement ends when the kettlebell is in front of and not above the head. Therefore, the rectus abdominis muscle would be less important to decelerate and stop the movement compared to the American swing. This is supported by muscle activation being far higher in the upper phase of the upward movement for the American (56$101 \%$ ) vs. Russian swing (12-19\%). Furthermore, ending the movement with one arm instead of two arms would impose more force on the shoulder joint. This would likely require a different motor control strategy to maintain the integrity of the joint, i. e., it could be more unsafe for the shoulder if the rectus abdominis muscle rapidly activated to decelerate the movement in the upper phase.

The lack of differences for the external oblique was in line with our previous study on the Russian swing [2] but not with previous strength exercise studies on the squat, row, and shoulder press $[1,3,16-18]$. These studies examined more traditional exercises and generally showed that unilateral execution increased the trunk muscle activation on the contralateral side compared to bilateral execution. However, the situation with traditional exercises vs. kettlebell exercise is somewhat different. In most traditional exercises, the trunk muscles are mainly proposed to stabilize statically the trunk to counteract lateral flexion or rotation due to the imbalance from the load being held outside the centerline of the body throughout the whole movement. This is not so much the case in kettlebell swing, where the kettlebell mostly is held in the centerline of the trunk, reducing the stress on the external obliques.

There are some limitations to consider. Surface EMG gives only an estimate of the neuromuscular activation and there will always be a possible risk of crosstalk from nearby muscles [8]. The EMG data was also collected during dynamic contractions, which have more potential sources for error than isometric contractions [8].
Importantly, all EMG data was collected in the same session, which substantially reduces the potential for error associated with electrode placement [14]. Further, only healthy men with little kettlebell experience were recruited to the study, and the results can therefore not necessarily be generalized to other populations. However, it should be emphasized that all participants conducted several practice sessions with a kettlebell instructor who approved their technique before performing the experimental session. Finally, a submaximal load (14-kg kettlebell) was used, and we cannot exclude the possibility that activation patterns could differ with other loads. Using the same absolute load (rather than relative) could be considered a limitation when comparing EMG data across exercises. The reason for using a 14-kg kettlebell in both exercises was that the prime mover (hip extensors) should not be affected by the use of one or two hands. Importantly, because the participants were a relatively homogenous group of resistance-trained men, the effort was considered moderate and similar between exercises (assessed with the Borg CR10-scale ${ }^{\circledR}$ ), we therefore expect this to have little impact on the findings.

In conclusion, the unilateral American kettlebell swing induced the highest activation of the erector spinae muscle on the contralateral side, at the expense of the ipsilateral side, as expected. However, bilateral execution induced the highest rectus abdominis muscle activation, whereas there were no differences for the external oblique muscle. These findings suggest that whether the American kettlebell swing is performed unilaterally or bilaterally should depend on which muscle of the trunk that requires emphasis. For the erector spinae muscle, unilateral execution could be most advisable, because the highest muscle activation was observed in the contralateral side. However, because the activation was reduced on the ipsilateral side, the same number of sets should be performed with each arm to provide the same stimuli on both sides. For the rectus abdominis muscle, the bilateral American kettlebell swing was clearly superior. For general health and fitness, it would be advisable to vary between the exercises over time.

\section{Acknowledgements}

We would like to thank Georg-Andre Jungård, Øyvind Vraalsen, Roy-Andrè Slåttland and Aril Gunnarskog along with the participants for assistance, positivity and participation in the study. This study was conducted without any funding from companies or manufacturers or outside organizations.

\section{Conflict of Interest}

There is no conflict of interest. This study was conducted without any funding from companies or manufacturers or outside organizations.

\section{References}

[1] Andersen V, Fimland MS, Brennset O, Haslestad LR, Lundteigen MS, Skalleberg K, Saeterbakken AH. Muscle activation and strength in squat and Bulgarian squat on stable and unstable surface. Int J Sports Med 2014; 35: 1196-1202 
[2] Andersen V, Fimland MS, Gunnarskog A, Jungard GA, Slattland RA, Vraalsen OF, Saeterbakken AH. Core muscle activation in one-armed and two-armed kettlebell swing. J Strength Cond Res 2016; 30: 1196-1204

[3] Behm DG, Leonard AM, Young WB, Bonsey WA, MacKinnon SN. Trunk muscle electromyographic activity with unstable and unilateral exercises. J Strength Cond Res 2005; 19: 193-201

[4] Brumitt J, En Gilpin H, Brunette M, Meira EP. Incorporating kettlebells into a lower extremity sports rehabilitation program. N Am J Sports Phys Ther 2010; 5: 257-265

[5] Bullock GS, Schmitt AC, Shutt JM, Cook G, Butler RJ. Kinematic and kinetic variables differ between kettlebell swing styles. Int J Sports Phys Ther 2017; 12: 324-332

[6] Cohen J. Statistical Power Analysis for the Behavioral Sciences. 2nd ed Hillsdale, N.J: L. Erlbaum Associates; 1988

[7] Falatic JA, Plato PA, Holder C, Finch D, Han K, Cisar C]. Effects of kettlebell training on aerobic capacity. J Strength Cond Res 2015; 29: 1943-1947

[8] Farina D. Interpretation of the surface electromyogram in dynamic contractions. Exerc Sport Sci Rev 2006; 34: 121-127

[9] Harriss D], Macsween A, Atkinson G. Standards for ethics in sport and exercise science research: 2018 update. Int J Sports Med 2017; 38: 1126-1131

[10] Hermens HJ, Freriks B, Disselhorst-Klug C, Rau G. Development of recommendations for SEMG sensors and sensor placement procedures. J Electromyogr Kinesiol 2000; 10: 361-374
[11] Lake JP, Lauder MA. Kettlebell swing training improves maximal and explosive strength. J Strength Cond Res 2012; 26: 2228-2233

[12] Lake JP, Lauder MA. Mechanical demands of kettlebell swing exercise. J Strength Cond Res 2012; 26: 3209-3216

[13] Manocchia P, Spierer DK, Lufkin AK, Minichiello J, Castro J. Transference of kettlebell training to strength, power, and endurance. J Strength Cond Res 2013; 27: 477-484

[14] Mathiassen SE, Winkel J, Hagg GM. Normalization of surface EMG amplitude from the upper trapezius muscle in ergonomic studies - a review. J Electromyogr Kinesiol 1995; 5: 197-226

[15] Otto WH 3rd, Coburn JW, Brown LE, Spiering BA. Effects of weightlifting vs. kettlebell training on vertical jump, strength, and body composition. J Strength Cond Res 2012; 26: 1199-1202

[16] Saeterbakken A, Andersen V, Brudeseth A, Lund H, Fimland MS. The effect of performing bi- and unilateral row exercises on core muscle activation. Int J Sports Med 2015; 36: 900-905

[17] Saeterbakken AH, Fimland MS. Muscle activity of the core during bilateral, unilateral, seated and standing resistance exercise. Eur J Appl Physiol 2012; 112: 1671-1678

[18] Santana JC, Vera-Garcia F], McGill SM. A kinetic and electromyographic comparison of the standing cable press and bench press. J Strength Cond Res 2007; 21: 1271-1277

[19] Tse MA, McManus AM, Masters RS. Development and validation of a core endurance intervention program: Implications for performance in college-age rowers. J Strength Cond Res 2005; 19: 547-552 